\title{
Algorithm Selection on Data Streams
}

\author{
Jan N. van Rijn ${ }^{1}$, Geoffrey Holmes ${ }^{2}$, \\ Bernhard Pfahringer ${ }^{2}$, and Joaquin Vanschoren ${ }^{3}$ \\ 1 Leiden University, Leiden, Netherlands, \\ j.n.van.rijn@liacs.leidenuniv.nl \\ 2 University of Waikato, Hamilton, New Zealand, \\ \{geoff, bernhard\}@cs. waikato.ac.nz \\ 3 Eindhoven University of Technology, Eindhoven, Netherlands, \\ j.vanschoren@tue.nl
}

\begin{abstract}
We explore the possibilities of meta-learning on data streams, in particular algorithm selection. In a first experiment we calculate the characteristics of a small sample of a data stream, and try to predict which classifier performs best on the entire stream. This yields promising results and interesting patterns. In a second experiment, we build a meta-classifier that predicts, based on measurable data characteristics in a window of the data stream, the best classifier for the next window. The results show that this meta-algorithm is very competitive with state of the art ensembles, such as OzaBag, OzaBoost and Leveraged Bagging. The results of all experiments are made publicly available in an online experiment database, for the purpose of verifiability, reproducibility and generalizability.
\end{abstract}

Keywords: Meta Learning, Data Stream Mining

\section{Introduction}

Modern society produces vast amounts of data coming, for instance, from sensor networks and various text sources on the internet. Various machine learning algorithms are able to capture general trends and make predictions for future observations with a reasonable success rate. The number of algorithms is large, and most of these work well on a varying range of data streams. However, there is not much knowledge yet about on which kinds of data certain algorithms perform well and when a certain algorithm should be preferred over another.

In this work we investigate how to predict what algorithm will perform well on a given data stream. This problem is generally known as the algorithm selection problem [14]. For each data stream, we measure the performance of various data stream classifiers and we calculate measurable data characteristics, called meta-features. In addition to many existing meta-features, we introduce a new type of meta-feature, based on concept drift detection methods. Next, we build a model that predicts which algorithms will work well on a given data stream based on its characteristics. Indeed, having knowledge about which classifier to apply on what data could greatly increase the performance of predictive tools in 
real world applications. For example, it is common for the performance curves of data stream algorithms to cross as the stream evolves. This means that at certain points in the stream, the data is best modelled with algorithm A, while at a later point, the data is better modelled with algorithm B. As such, selecting the right algorithm at each point in the stream has the potential to increase performance. In this work we focus on data streams with a nominal target. Although much work has been done on both data streams and meta-learning, to the best of our knowledge, this is the first effort to do meta-learning on data streams.

The remainder of this paper is structured as follows. Section 2 contains a description of related work. Section 3 describes how the meta-dataset was created, the data streams that it contains, how they are characterized, and how we have measured the performance of data stream algorithms. Section 4 describes an experiment in which we calculate the characteristics of a small sample of a data stream, and predict which classifier performs best on the entire stream. Next, Section 5 describes a second experiment, in which we continuously measure the characteristics of a sliding window on the data stream, and use a meta-algorithm to predict the best classifier for the next window. In Section 6 we present and discuss some emerging patterns from the data. Section 7 concludes.

\section{Related Work}

It has been recognized that mining data streams differs from conventional batch data mining $[4,13]$. In the conventional batch setting, usually a limited amount of data is provided and the goal is to build a model that fits the data as well as possible, whereas in the data stream setting, there is a possibly infinite amount of data, with concepts possibly changing over time, and the goal is to build a model that captures general trends.

More specifically, the requirements for processing streams of data are: process one example at a time (and inspect it only once), use a limited amount of time and memory, and be ready to predict at any point in the stream $[3,13]$. These requirements inhibit the use of most batch data mining algorithms. However, some algorithms can trivially be used or adapted to be used in a data stream setting, for example, NaiveBayes, $k$ Nearest Neighbour, and Stochastic Gradient Descent, as done in [13]. Also, many algorithms have been created specifically to operate on data streams. Most notably, the Hoeffding Tree [6] is a tree based algorithm that splits based on information gain, but using only a small sample of the data determined by the Hoeffding bound. The Hoeffding bound gives an upper bound on the difference between the mean of a variable estimated after a number of observations and the true mean, with a certain probability.

Conventional batch data mining methods can also be adapted for use in the data streams setting by training them on a set of instances sampled from recent data. Typically, a set of $w$ (window size) training instances is formed. Every $w$ instances form a batch and are provided to the learner, which builds a model based on these instances. The disadvantages of this approach are that the most recent examples are not used until a batch is complete, and old models need to 
be deleted to make room for new models. Read et al. [13] distinguish between instance incremental methods and batch incremental methods and compare the performance of both approaches. Their main conclusion is that the performance in terms of accuracy is equivalent. However, the instance incremental algorithms use fewer resources.

Ensembles of classifiers are among the best performing learning algorithms in the traditional batch setting. Multiple models are produced that all vote for the label of a certain instance. The final prediction is made according to a predefined voting schema, e.g., the class with the most votes wins. In [10] it is proven that the error rate of an ensemble in the limit goes to zero if two conditions are met: first, the individual models must do better than random guessing, and second, the individual models must be diverse, meaning that their errors should not be correlated. Popular ensemble methods in the traditional batch setting are Bagging [5], Boosting [17] and Stacking [7]. Bagging and Boosting have equivalents in the data stream setting, e.g., OzaBag [11], OzaBoost [11] and Leveraging Bagging [4]. The Average Weighted Ensemble [20] tracks which individual classifiers perform well on recent data, and uses this information to weight the votes.

The field of meta-learning addresses the question what machine learning algorithms work well on what data. The algorithm selection problem, formalised by Rice in [14], is a natural problem from the field of meta-learning. According to the definition of Rice, the problem space $P$ consists of all machine learning tasks from a certain domain, the feature space $F$ contains measurable characteristics calculated upon this data (called meta-features), the algorithm space $A$ is the set of all considered algorithms that can execute these tasks and the performance space $Y$ represents the mapping of these algorithms to a set of performance measures. The task is for any given $x \in P$, to select the algorithm $\alpha \in A$ that maximizes a predefined performance measure $y \in Y$, which is a classification problem. Similar ranking and regression problems are derived from this.

Much effort has been devoted to the development of meta-features that effectively describe the characteristics of the data (called meta-features). Commonly used meta-features are typically categorised as one of the following: simple (number of instances, number of attributes, number of classes), statistical (mean standard deviation of attributes, mean kurtosis of attributes, mean skewness of attributes), information theoretic (class entropy, mean entropy of attributes, noise-signal ratio) or landmarkers [12] (performance of a simple classifier on the data). The authors of [18] give an extensive description of many meta-features. Furthermore, they propose a new type of meta-feature, pair-wise meta-rules.

Another recent development is the concept of experiment databases $[15,19]$, databases which contain detailed information about a large range of experiments. Experiment databases enable the reproduction of earlier results for verification and reusability purposes, and make much larger studies (covering more algorithms and parameter settings) feasible [19]. Above all, experiment databases allow a variety of studies to be executed by a database look-up, rather than setting up new experiments. An example of such an online experiment database is OpenML [15]. 


\section{Meta-dataset}

For the purpose of meta-learning, we need to obtain data on previous experiments, i.e., runs of various algorithms on various data streams (from here on referred to as base datasets, or base data streams). From this we construct a socalled meta-dataset, in which each instance is a data stream characterized by a number of measurable characteristics, the meta-features, as well as performance scores of various machine learning algorithms.

\subsection{Bayesian Network Generator}

Unfortunately, the data stream literature contains few publicly available data streams. In order to obtain a reasonable number of experiments on data streams, we propose a new type of data generator that generates data streams based on real world data [16]. It takes a dataset as input, preferably consisting of real world data and a reasonable number of features, and builds a Bayesian Network over it, which is then used to generate instances based on the probability tables. These streams can also be combined together to simulate concept drift, similar to what is commonly done with the Covertype, Pokerhand and Electricity dataset [4].

The generator takes a dataset as input, and outputs a data stream containing a similar concept, with a predefined number of instances. The input dataset is preprocessed with the following operations: all missing values are first replaced by the majority value of that attribute, and numeric attributes are discretized using Weka's binning algorithm [9]. Values for attributes that are numeric in the original dataset can be determined using two strategies. The nominal strategy assigns one of the bins as the attribute value, determining the bin based on the probability tables. The numeric strategy takes the bin with the highest probability value and draws a number from this bin based on its normal distribution. The generated data streams are denoted as BNG(data, strategy, num_instances), with data denoting the original dataset, strategy denoting the chosen strategy for numeric values, and instances denoting the number of generated instances. The algorithm is implemented in the OpenML MOA package ${ }^{4}$.

Figure 1 shows the meta-features as calculated over the two Bayesian Network Generated data streams based on the glass dataset, compared to the values of the original dataset. As many of these qualities are quite similar, there is some indication that there is a similar concept underlying the data. The dimensionality indicates the ratio between the number of instances and the number of attributes. The decrease of this value can be explained by the fact that the number of attributes is the same, yet the number of instances has increased. Furthermore, data streams generated using the nominal strategy do not have any numeric attributes, hence meta-features like Mean Skewness Of Numeric Attributes and Mean StDev Of Numeric Attributes are zero. The meta-features indicating the J48 landmarkers (with varying confidence factors) have better values on the generated data streams, hinting at a slightly easier concept represented by the data. Similar patterns can be found for other generated data streams.

\footnotetext{
${ }^{4}$ can be obtained from http://www. openml.org/
} 


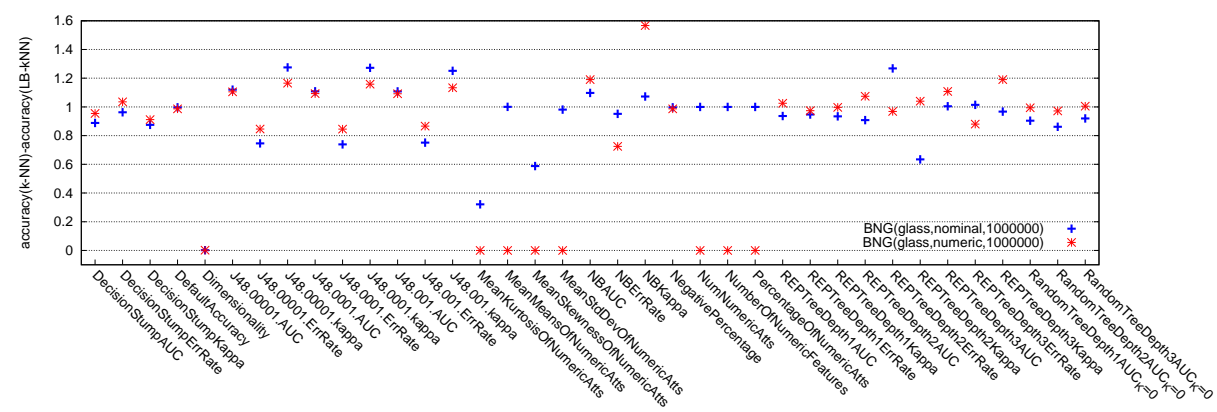

Fig. 1. Meta-features of two Bayesian Network Generated data streams, where each value is divided by the corresponding meta-feature value of the original dataset.

\subsection{Base data streams}

The data streams generated by the Bayesian Network Generator form the basis of our meta-dataset. We took datasets from the UCI repository [1] as input for the Bayesian Network generator. We aimed to generate data streams of 1,000,000 instances, or less if the original dataset did not have enough attributes to obtain a million different instances. We used both the nominal and the numeric strategy in those cases where the original dataset had numeric attributes. In addition to these, we also generate data streams by commonly used data generators from the literature. We used the following data generators, as implemented in the MOA workbench [3]: the SEA Concepts Generator, Rotating Hyperplane Generator, Random RBF Generator and the LED Generator. For the generation of these data streams we used the same parameters as described in [13].

Additionally, we also included the commonly used datasets Covertype, Electricity and Pokerhand, and a combination of these in order to generate concept drift, as is done in [4]. We also included large text datasets, i.e., the IMDB dataset and the 20 Newsgroups dataset. We converted the IMDB dataset into a binary classification problem, having the drama genre as target. The 20 Newsgroups dataset is first converted into 20 binary classification problems (one for every Newsgroup) and then appended again into one big binary-class dataset, as is done in [13]. This simulates a data stream with 19 concept drifts.

\subsection{Meta-features}

We have characterized the base data streams using a wide variety of metafeatures. Most of the features are already described in the literature, e.g., in [18], and are either of the type simple, statistical, information theoretic or landmarker. We also introduce stream specific meta-features, based on a change detector. We have run both Hoeffding Trees and NaiveBayes with both the ADWIN [2] and DDM [8] change detectors over all data streams, and have recorded the number 
Table 1. Algorithms used in the experiments. Batch incremental classifiers have a window of size 1,000; ensembles contain 10 base classifiers; $\mathrm{k}-\mathrm{NN}$ is used with $k=10$.

\begin{tabular}{lll}
\hline Key & Classifier & Type \\
\hline NB & NaiveBayes & Instance incremental \\
SGD & Stochastic Gradient Descent & Instance incremental \\
SPeg & SPegasus & Instance incremental \\
k-NN & $k$ Nearest Neighbour & Instance incremental \\
HT & Hoeffding Tree & Instance incremental \\
SMO & Support Vector Machine / Polynomial Kernel & Batch incremental \\
J48 & C4.5 Decision Tree & Batch incremental \\
REP & Reduced-Error Pruning Decision Tree & Batch incremental \\
OneR & One Rule & Batch incremental \\
LB-kNN & Leveraging Bagging / k-NN & Ensemble \\
LB-HT & Leveraging Bagging / Hoeffding Tree & Ensemble \\
Bag-HT & OzaBag / Hoeffding Tree & Ensemble \\
Boost-HT & OzaBoost / Hoeffding Tree & Ensemble \\
\hline
\end{tabular}

of changes detected (both ADWIN and DDM) and the number of warnings (DDM only). All these meta-features are calculated for each window of 1,000 instances on each data stream.

\subsection{Algorithms}

The algorithms included in this study are shown in Table 1, and can be grouped into three types: instance incremental classifiers, batch incremental classifiers and ensembles. For all classifiers, we have recorded the predictive accuracy, the runtime, and RAM Hours on each data stream. The predictive accuracy was determined using the Interleaved Test Then Train procedure, where each instance is first used as a test instance, before it can be used to train the classifier.

\section{Algorithm Selection in the Classical Setting}

In the classical setting of the algorithm selection problem, the goal is to predict, for a certain dataset, what algorithm would perform best.

\subsection{Experimental Setup}

In prior studies, the algorithm selection problem was treated as either a classification problem, regression problem, or ranking problem [18]. To investigate what kind of information can be obtained from the data, we treat the algorithm prediction problem on data streams as a classification problem. We create a meta-dataset ${ }^{5}$ using the experimental data described in Section 3. For each base

\footnotetext{
${ }^{5}$ All meta-datasets that were created for this study can be obtained from http://www.openml.org/d/
} 
Table 2. Results of the algorithm selection problems in the classical setting.

\begin{tabular}{|c|c|c|c|c|c|c|c|}
\hline Task & $A$ & Majority & $\%$ & Decision & Stump & Random & Forest \\
\hline Instance incremental & 5 & HT & 71.25 & & 79.63 & & 88.46 \\
\hline Batch incremental & 4 & SMO & 67.86 & & 65.07 & & 68.73 \\
\hline Ensembles & 4 & LB-HT & 62.25 & & 63.98 & & 67.11 \\
\hline All classifiers & 13 & LB-HT & 61.43 & & 60.14 & & 69.57 \\
\hline
\end{tabular}

data stream, the meta-features are recorded in the first window of 1,000 observations. By the definition of Rice [14], this means that we are working on the algorithm selection problem with $A=13$ (algorithms), $P=75$ (data streams), $Y=$ predictive accuracy (performance measure) and $F=58$ (meta-features).

The goal is to predict which algorithm performs best, measured over the whole data stream. In order to obtain deeper insight into what kind of targets we can predict, we also defined three sub tasks, i.e., predicting the best instance incremental classifier $(A=5)$, predicting the best batch incremental classifier $(A=4)$ and predicting the best ensemble $(A=4)$. We have selected the "Decision Stump" and "Random Forest" classifiers (as implemented in Weka 3.7.11 [9]) as meta-algorithms. The Random Forest algorithm has proven to be a useful meta-algorithm in prior work [18], while models obtained from a single decision tree or stump are especially easy to interpret. Both classifiers are tree-based, which guards them against modeling irrelevant features. We ran the Random Forest algorithm with 100 trees and 10 attributes. We estimate the performance of the meta-algorithm by doing 10 times 10 fold cross-validation, and compare its performance against predicting the majority class. For each metadataset, we have filtered out the instances that contain a unique class value; since they will either only appear in the training or test set, these do not form a reliable source for estimating the accuracy.

\subsection{Results}

Table 2 shows the results obtained from the various tasks. It shows for every task which classifier performs best in most cases (and therefore is the majority class of the task) and in what percentage of the data streams that is the case. Column " $A$ " denotes the number of classes that were distinguished in the tasks; column "Majority" and "\%" denote the majority class and its size. The other columns denote the accuracy score of the respective meta-algorithms. The Random Forest algorithm performs better than the baseline on all defined tasks. The Decision Stump algorithm performs well only at predicting both the best instance incremental classifiers and ensembles. The results of both metaalgorithms on the task of predicting the best instance incremental were marked as significantly better than predicting majority class, tested using a Paired TTest with a confidence of $95 \%$. Since the data characteristics were obtained over only the first 1,000 instances of each stream, algorithm selection on data streams can improve results of classifiers at already very low computational cost. 


\section{Algorithm Selection in the Stream Setting}

In data stream prediction, it is common for performance curves of different algorithms to cross each other multiple times. Whereas, at a certain interval of the stream, one of the algorithms performs best, this might be different for other intervals of the stream. By applying algorithm selection in a stream setting, our goal is to predict what algorithm will perform best for the next window of data.

\subsection{Experimental Setup}

In this experiment we want to determine whether meta-knowledge can improve the predictive performance of data stream algorithms in the following setting. Consider an ensemble of algorithms that are all trained on the same data stream. For each window of size $w$, an abstract meta-algorithm determines which algorithm will be used to predict the next window of instances, based on data characteristics measured in the previous window and the meta-knowledge. Note that the performance of the meta-algorithm depends on the size of this window. Meta-features calculated over a small window size are probably not able to adequately represent the characteristics of the data, whereas calculating metafeatures over large windows is computationally expensive. Since our previous experiment obtained good results with a window size of 1,000 , we perform our experiments with the same window size.

We have constructed a new meta-dataset, containing for each window the meta-features measured over the previous interval, and the performance of all base-classifiers trained on the entire data stream up to that window. We only include a subset of the generated data streams. Indeed, since the Bayesian Network Generator was used to generate multiple data streams based upon the same source data (using the nominal strategy and the numeric strategy), it is likely that these data streams contain a similar concept. We therefore remove all data streams generated using the nominal strategy, if a version generated by the numeric strategy also exists. After filtering out these data streams, there are still 49 data streams left. The meta-dataset consists of roughly 45,000 instances, each describing a window of 1,000 observations from one of these base data streams.

For each of the base data streams, a meta-algorithm is trained using only the intervals of the other data streams. We use a Random Forest (100 trees, 10 attributes) as meta-algorithm, since it proved to be a reasonable choice in

the previous experiment. We measure how it performs on the meta-learning task of predicting the right algorithm for a given interval, as well as how it would actually perform on the base data streams. To the best of our knowledge, Leveraged Bagging Hoeffding Trees is the state of the art algorithm on these data streams, so we will compare it against our abstract algorithm. As in the previous experiment, we distinguish the tasks of selecting the best instance incremental classifier, the best batch incremental classifier and the best ensemble. 
Table 3. Results of the algorithm selection problems in the stream setting.

\begin{tabular}{lrlrrrrr}
\hline Task & $A$ & Majority & $\%$ & $\mathrm{RF}_{\text {meta }}$ & ZeroR $_{\text {base }}$ & $\mathrm{RF}_{\text {base }}$ & $\mathrm{MAX}_{\text {base }}$ \\
\hline Instance incremental & 5 & HT & 59.75 & 80.78 & 80.98 & 84.07 & 84.59 \\
Batch incremental & 4 & SMO & 65.56 & 68.17 & 74.38 & 75.33 & 76.02 \\
Ensembles & 4 & LB-HT & 57.78 & 56.20 & 84.27 & 85.15 & 86.12 \\
All classifiers & 13 & LB-HT & 50.97 & 50.92 & 84.27 & 85.31 & 86.30 \\
\hline
\end{tabular}

\subsection{Results}

Table 3 shows the results obtained from this experiment. As with the results of the previous experiment, column $A$ indicates the number of classes in the classification problem, "Majority" denotes which classifier is the majority class (i.e., the classifier that performs best in most observations), and "\%" shows the size of the majority class. We measure two types of accuracy, meta-level accuracy and base-level accuracy. Meta-level accuracy records the performance using a zero-one loss function. Consequently, it indicates for how many windows the meta-algorithm predicted the best classifier. Column $\mathrm{RF}_{\text {meta }}$ shows the metalevel accuracy of a Random Forest. Base-level accuracy records the performance using a loss function equivalent to the performance of the predicted classifier. For example, when the meta-algorithm predicts $\mathrm{k}-\mathrm{NN}$ to be the best classifier on a certain interval, the accuracy of $\mathrm{k}$-NN on this interval will be used as loss. Accordingly, base-level accuracy indicates the performance of the meta-classifier on the base data streams when dynamically selecting the base-classifier. The base-level score of the Random Forest meta-algorithm is shown in column $\mathrm{RF}_{\text {base }}$. Column Zero $_{\text {base }}$ shows the base-level accuracy when the majority class is always predicted. Note that this is the score obtained by the majority class base-classifier, measured over all base data streams. Column MAX base $_{\text {shows what the base-level }}$ score would be, if for any interval the best classifier would have been predicted.

As in the classical setting, it appears that determining the best instanceincremental classifier yields good results. In more than $80 \%$ of the cases, the correct classifier is predicted. This also results in a notable increase in base level performance, in such a way that it is comparable with Leveraged Bagged Hoeffding Trees (84.27), and outperforms the scores obtained by OzaBag (82.58) and OzaBoost (80.55). The results also show a consistent increase in performance. For all defined tasks, the meta-algorithm outperforms the use of the single best classifier in its pool, even though, on the ensemble task, $R F_{\text {meta }}$ performs no better than predicting the majority class. Apparently, the metaalgorithm was able to avoid the use of Leveraged Bagged Hoeffding Trees on windows where the performance is very low. Furthermore, the base level performances are in many cases close to the maximum possible value given the pool of classifiers. This indicates that the main challenge is to find ways to improve this limit. This could be done by using a larger set of algorithms, or by using other techniques such as parameter optimisation. 


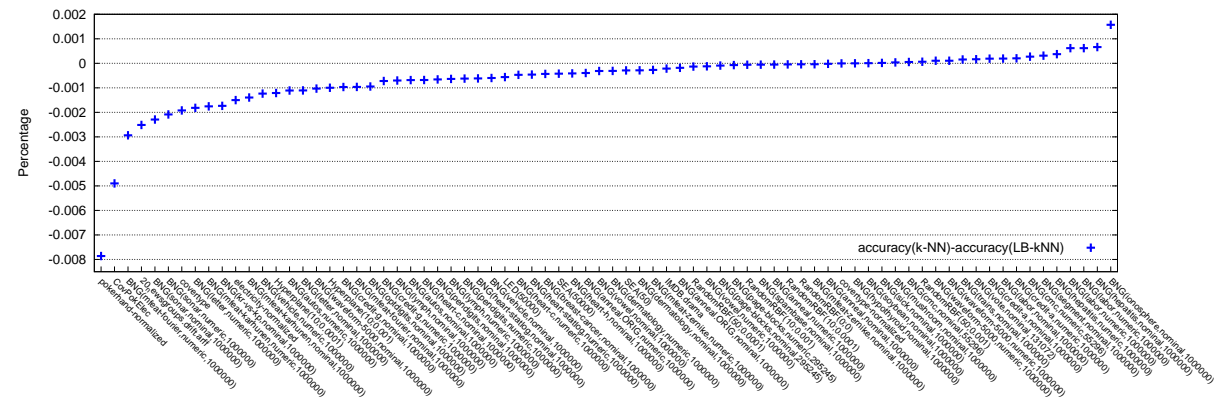

Fig. 2. The difference in predictive accuracy of $k$-NN and Leveraged Bagging $k$-NN. $(k=10,10$ base classifiers)

\section{Discoveries}

In this section we discuss interesting patterns that were obtained while analysing the meta-datasets, some of which corroborate earlier findings in the literature. Other findings will form the basis of our future work.

Discovery 1 An abstract meta-classifier consisting of 5 instance incremental classifiers (NaiveBayes, 10 Nearest Neighbour, SPegasus, SGD and a Hoeffding Tree) is competitive with state of the art ensembles, tested over a large range of data streams. It is likely that this result generalizes to an even larger number of data streams, because both conditions for an ensemble of classifiers to be successful are met [10]: the base classifiers are diverse and do better than random guessing. Moreover, the experiment in Section 4 shows that it is possible to predict which one will perform well based on prior data.

Discovery 2 In contrast to the increase in performance that Leveraging Bagging obtains when applied to Hoeffding Trees, it barely increases performance when applied to $k$ Nearest Neighbour. Figure 2 shows the difference in performance of both algorithms. Note that, although Leveraged Bagging $k$-NN performs slightly better, the difference is minuscule. This may be due to the fact that even in a stream setting, $k$-NN is extremely stable; Bagging exploits the variation in the predictions of classifiers. In [5], it was already shown that Bagging will not improve $k$-NN in the batch setting, due to its stability.

Discovery 3 The data streams on which $k$-NN or Leveraging Bagging $k$-NN performs best all have a negative Mean Skewness of attributes. Skewness is a measure of the asymmetry in the distribution of a range of values. A negative Skewness indicates that there are some outliers with low values. This was already observed in the first interval of 1,000 instances, the Decision Stump extensively uses this feature to distinguish between predicting $k$-NN and Hoeffding 
trees. This is surprising, since it has been reported that simple, statistical and information theoretic meta-features do not add much predictive power to landmarkers [18].

Discovery 4 NaiveBayes works well when the meta-feature measuring the number of changes detected by an ADWIN-equipped Hoeffding Tree has a high value. The NaiveBayes algorithm generally needs only relatively few observations to achieve good accuracy compared to more sophisticated algorithms such as Hoeffding Trees. Assuming that a high number of changes detected by this landmarker indicates that the concept of the stream is indeed changing quickly, this could explain why a classifier like NaiveBayes outperforms more sophisticated learning algorithms that need more observations of the same concept to perform well.

\section{Conclusions}

We have performed an extensive experiment on meta-learning on data streams, running a wide range of steam mining algorithms over a large number of data streams, and published all results online in OpenML [15], so that others can verify, reproduce and build upon these results. Containing more than 1,000 experiments on data streams, with extensive meta-information calculated over data (windows), this now forms a rich source of meta-learning experiments on data streams. In order to obtain a good number of data streams, the Bayesian Network Generator was introduced, a new data stream generator used to generate a comprehensive set of data streams describing various concepts.

Our approach to perform meta-learning on these streams seems promising: Meta-features calculated on a small interval at the start of the data stream already provide information about which classifier will outperform others. Beyond the classical setting of the algorithm selection problem, we can even use the meta-models obtained from earlier experiments to improve the current state of the art classifiers. We have sketched an abstract algorithm that uses multiple classifiers and a voting schema based on meta-models that outperforms the performance of the individual classifiers in its ensemble, but also is very competitive with state of the art ensembles, measured over 49 data streams spanning more that 46,000,000 instances. In addition, we discussed interesting patterns that emerged from the meta-dataset, which will form a basis for future work.

Moreover, in this work we have treated the algorithm selection problem as a classification task. In future work we will focus on ranking or regression tasks. We will also include more data streams containing concept drift, and study the effect on classifier performance. Due to the use of data generators this study may potentially be biased towards this kind of generated data. We hope that making our meta-dataset publicly available will persuade others to share more data streams, eventually enabling much larger studies on even more diverse data.

Acknowledgments This work is supported by grant 600.065 .120 .12 N150 from the Dutch Fund for Scientific Research (NWO). 


\section{References}

1. Bache, K., Lichman, M.: UCI machine learning repository (2013), http:// archive.ics.uci.edu/ml

2. Bifet, A., Gavalda, R.: Learning from Time-Changing Data with Adaptive Windowing. In: SDM. vol. 7, pp. 139-148. SIAM (2007)

3. Bifet, A., Holmes, G., Kirkby, R., Pfahringer, B.: MOA: Massive Online Analysis. J. Mach. Learn. Res. 11, 1601-1604 (2010)

4. Bifet, A., Holmes, G., Pfahringer, B.: Leveraging Bagging for Evolving Data Streams. In: Machine Learning and Knowledge Discovery in Databases, Lecture Notes in Computer Science, vol. 6321, pp. 135-150. Springer (2010)

5. Breiman, L.: Bagging Predictors. Machine learning 24(2), 123-140 (1996)

6. Domingos, P., Hulten, G.: Mining high-speed data streams. In: Proceedings of the sixth ACM SIGKDD international conference on Knowledge discovery and data mining. pp. $71-80(2000)$

7. Gama, J., Brazdil, P.: Cascade Generalization. Machine Learning 41(3), 315-343 (2000)

8. Gama, J., Medas, P., Castillo, G., Rodrigues, P.: Learning with Drift Detection. In: SBIA Brazilian Symposium on Artificial Intelligence, Lecture Notes in Computer Science, vol. 3171, pp. 286-295. Springer (2004)

9. Hall, M., Frank, E., Holmes, G., Pfahringer, B., Reutemann, P., Witten, I.H.: The WEKA Data Mining Software: An Update. ACM SIGKDD explorations newsletter 11(1), 10-18 (2009)

10. Hansen, L., Salamon, P.: Neural network ensembles. Pattern Analysis and Machine Intelligence, IEEE Transactions on 12(10), 993-1001 (1990)

11. Oza, N.C.: Online Bagging and Boosting. In: Systems, man and cybernetics, 2005 IEEE international conference on. vol. 3, pp. 2340-2345. IEEE (2005)

12. Pfahringer, B., Bensusan, H., Giraud-Carrier, C.: Tell me who can learn you and I can tell you who you are: Landmarking various learning algorithms. In: Proceedings of the 17th international conference on machine learning. pp. 743-750 (2000)

13. Read, J., Bifet, A., Pfahringer, B., Holmes, G.: Batch-Incremental versus InstanceIncremental Learning in Dynamic and Evolving Data. In: Advances in Intelligent Data Analysis XI, pp. 313-323. Springer (2012)

14. Rice, J.R.: The Algorithm Selection Problem. Advances in Computers 15, 65118 (1976)

15. van Rijn, J.N., Bischl, B., Torgo, L., Gao, B., Umaashankar, V., Fischer, S., Winter, P., Wiswedel, B., Berthold, M.R., Vanschoren, J.: OpenML: A Collaborative Science Platform. In: Machine Learning and Knowledge Discovery in Databases, pp. 645-649. Springer (2013)

16. van Rijn, J.N., Holmes, G., Pfahringer, B., Vanschoren, J.: The Bayesian Network Generator: A data stream generator. Tech. Rep. 03/2014, Computer Science Department, University of Waikato (2014)

17. Schapire, R.E.: The Strength of Weak Learnability. Machine learning 5(2), 197-227 (1990)

18. Sun, Q., Pfahringer, B.: Pairwise meta-rules for better meta-learning-based algorithm ranking. Machine learning 93(1), 141-161 (2013)

19. Vanschoren, J., Blockeel, H., Pfahringer, B., Holmes, G.: Experiment databases. A new way to share, organize and learn from experiments. Machine Learning 87(2), 127-158 (2012)

20. Wang, H., Fan, W., Yu, P.S., Han, J.: Mining Concept-Drifting Data Streams using Ensemble Classifiers. In: KDD. pp. 226-235 (2003) 